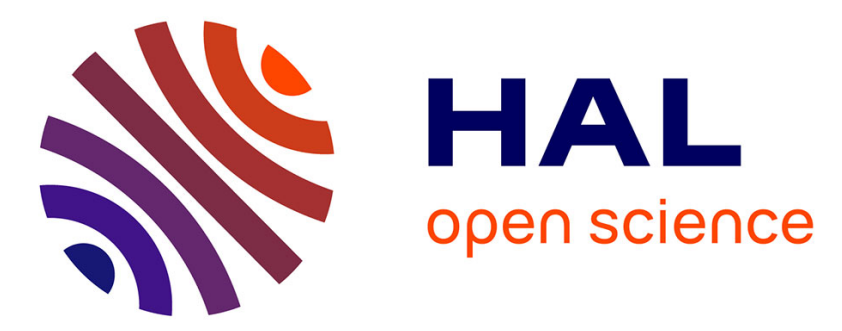

\title{
An Energy-aware End-to-End Crowdsensing Platform: Sensarena
}

\author{
Rim Ben Messaoud, Zeineb Rejiba, Yacine Ghamri-Doudane
}

\section{To cite this version:}

Rim Ben Messaoud, Zeineb Rejiba, Yacine Ghamri-Doudane. An Energy-aware End-to-End Crowdsensing Platform: Sensarena. IEEE Consumer Communications \& Networking Conference (CCNC), Jan 2016, Las-Vegas, United States. hal-01287714

\section{HAL Id: hal-01287714 https://hal.science/hal-01287714}

Submitted on 20 Mar 2016

HAL is a multi-disciplinary open access archive for the deposit and dissemination of scientific research documents, whether they are published or not. The documents may come from teaching and research institutions in France or abroad, or from public or private research centers.
L'archive ouverte pluridisciplinaire $\mathbf{H A L}$, est destinée au dépôt et à la diffusion de documents scientifiques de niveau recherche, publiés ou non, émanant des établissements d'enseignement et de recherche français ou étrangers, des laboratoires publics ou privés. 


\title{
An Energy-aware End-to-End Crowdsensing Platform: Sensarena
}

\author{
Rim Ben Messaoud ${ }^{* \dagger}$, Zeineb Rejiba ${ }^{* \dagger}$ and Yacine Ghamri-Doudane ${ }^{\dagger}$ \\ *Université Paris-Est, Laboratory of Informatics Gaspard Monge (LIGM), Champs sur Marne, France \\ $\dagger$ University of La Rochelle, Laboratory of Informatics, Image and Interaction (L3I), La Rochelle, France \\ \{rim.ben_messaoud, yacine.ghamri\}@univ-lr.fr, zeineb.rejiba@gmail.com
}

\begin{abstract}
Nowadays, smart-devices come with a rich set of built-in sensors besides being full-fledged processing and communicating handsets. This empowers the crowd to collect and share sensed data about various city-related phenomena, a new paradigm denoted as Crowdsensing. In this context, we introduce Sensarena; an end-to-end general-use crowdsensing platform which consists of three main entities: two types of android-based mobile applications and a central server. The first application is destined to the participants to conduct sensing campaigns while the second is for requestors to submit their sensing requests. Besides, the server side is designed to host energy-aware sensing tasks assignment mechanisms and storage of different types of data. The developed platform has been exhaustively tested for different scenarios and proved a competitive performance while responding to both participants and requestors requirements.
\end{abstract}

\section{INTRODUCTION}

The proliferation of smart devices with embedded multiuse sensors enhances the role of the general public (Crowd) in data collection and sharing. Crowdsensing has attracted a lot of attention from both academia and industry thanks to its potential applications such as traffic monitoring [1], environment monitoring [2] and health care to name a few.

Among the various crowdsensing systems introduced in the literature, only few were general-use ones. For instance, Funf Open Sensing Framework ${ }^{1}$, is a generic tool for scientists to configure their search criteria to be built into a ready-to-use application destined to participants. Yet, the provided sensing apps are considered to be very basic which limits their fields of application. Another platform introduced by researchers is APISense $^{2}$. This Software as a Service (SaaS) solution models the requested sensing tasks as scripts which allows code portability across different mobile platforms. Additionally, a PRIVAPI model was added to the APISense platform to respond to participants' concerns about privacy. Nevertheless, for both aforementioned platforms, efficient task allocation schemes were not investigated which may result in a nonefficient sensing process in terms of dedicated resources.

Sensarena is then a crowdsensing platform that we introduce in this paper to tackle the different challenges of sensing deployment. Hence, we designed a tasks assignment process running on the server side to allocate sensing tasks according to the participants' preferences and requestors' demands. Besides, two types of android-based mobile applications were developed to allow users to receive and perform sensing requests as participants or submit sensing tasks as requestors.
In the remainder of this paper, we provide an overview of the Sensarena architecture and features in section II and we detail the demo description in section III before concluding.

\section{The Proposed Crowdsensing Platform: SENSARENA}

\section{A. Architecture}

The Sensarena tiered architecture, detailed in Figure 1, describes the main elements of a crowdsensing system. The presentation tier on the left represents the mobile devices and involves two types of user classes; the participants equipped with an android-based application, SensarenaP, that leverages the different built-in sensors in their handsets to collect data and the requestors provided with the SensarenaR application which enables them to look for sensor information. On the right, the business logic tier and the data tier are illustrated by a Node.js central server and a NoSQL database (MongoDB) respectively. The latter is organized into three main collections in order to store separately all user-related information, the sensing task details and the collected data.

\section{B. Sensarena Features}

Hereafter, we describe the different features of our energyaware general-use crowdsensing platform, Sensarena, while focusing on the proposed task assignment scheme and measures taken to address the energy constraint.

First, users need to subscribe to the platform. That is, they must provide their credentials via the Registration screen of the SensarenaP app. Once registered, users are automatically redirected to the Authentication screen to $\log$ in and start their crowdsensing experience. Note that a Preferences editing feature is provided here in order to ensure participants' commitment during the sensing process by allowing them to select which sensors' data to contribute.

Based on received users' coordinates and preferences, the central server launches the Task assignment process. This is performed periodically to look for newly submitted sensing tasks by requestors. A first selection considers all the participants registered within the area of interest, i.e., the users whose cities contain the corresponding sensing region. However, the final set of candidates of a specific task must include only users whose preferences conform with its requirements. Therefore, only adequate users will be notified about the available sensing requests while precising the location and the 


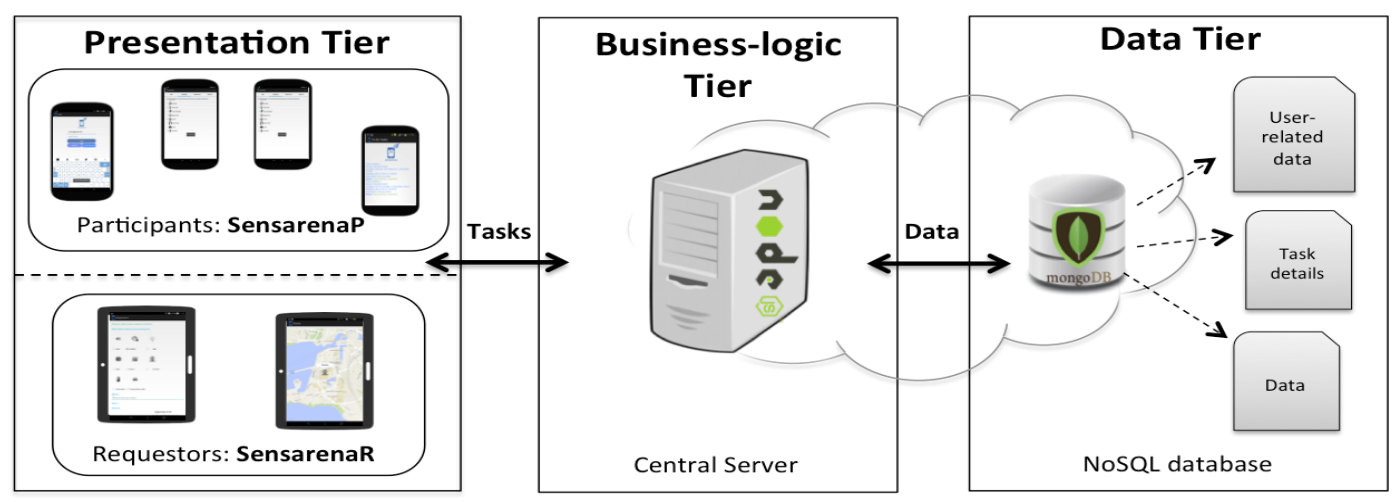

Fig. 1. Architecture and core features of the crowdsensing platform: Sensarena.

deadline. This avoids the overhead that may be generated when communicating the different entities of the Sensarena platform besides lowering the burden placed on participants.

After receiving notifications of the first assignment, users can accept or reject to participate to the announced sensing campaigns. By accepting, users report their actual positions and their handsets' battery level to the platform core. Next, the server conducts the Participants selection process. This feature partly inherits proposals from the QoI and Energyaware Mobile Selection Scheme, introduced by authors in [3], to include two main criteria. First, users' handsets must satisfy an energy constraint defined by a threshold, below which participation in sensing tasks is not allowed. This ensures to participants the necessary energetic resources for the common use of their mobile devices. The second criterion enhances the accuracy of the collected data samples by allocating the task in question to the closer participant(s) to the point of measurement. Thus, the final set of participants is selected among the best ranked users, based on the aforementioned criteria, and notified to start performing the sensing.

The Sensing execution and Data upload is launched by activating/deactivating the necessary sensors automatically and uploading the collected data to the server to be stored into the database for future use. This highly decreases the overall energy cost of the task execution and limits the participant's involvement during the sensing process. Later, the uploaded data can be retrieved by requestors via the Search sensed data feature built in their SensarenaR mobile application. The latter enables both search and submission of sensing tasks.

\section{DEMo Description}

The capabilities of the Sensarena platform are demonstrated by two use cases depending on the type of involved sensors:

Built-in mobile devices' sensors: As a first step, we exhibit the traditional use case of a requestor searching data about a phenomenon measured by sensors embedded in smart devices such as; barometer, accelerometer, microphone. If found, the measurement will be displayed on a map-screen of the SensarenaR android-based application. Else, the requestor needs to submit a new sensing task to the central server via the same application. The server launches accordingly its periodic assignment to find the adequate subset of users who may perform this task as detailed in section II. After conducting the necessary selection process and allocating tasks to participants, the server receives the required data and delivers it back to the requestor. Hence, Demo visitors would be able to define different sensing tasks with different sensed phenomena, periods and number of required samples, using the SensarenaR mobile application.

External sensors: The second step is to retrieve data from external sensors. In our work, we communicate the SensarenaP android-based application to an external device denoted as Sensortag $^{3}$ which allows measuring different environmental aspects such as pressure and temperature. Thus, users may conduct periodic measurements of such sensors data and upload it to the Sensarena platform. Consequently, when receiving a demand of a temperature measurement via the SensarenaR app, the server looks for already collected samples in its database and responds while taking into account the corresponding submitted search criteria.

\section{CONCLUSions}

In this paper, we presented Sensarena our energy-aware endto-end generic-use crowdsensing solution. The various features of the platform offered to both requestors and participants were identified. Besides, we introduced the two-step task allocation scheme to ensure a quality-aware and energy-efficient sensing process. Tests of different use cases proved a promising performance that is likely to allow a future large-scale adoption.

\section{REFERENCES}

[1] P. Mohan, V. N. Padmanabhan, and R. Ramjee, "Nericell: Rich monitoring of road and traffic conditions using mobile smartphones," in ACM conf. on Embedded Network Sensor Systems, 2008, pp. 323-336.

[2] M. Mun, S. Reddy, K. Shilton, N. Yau, J. Burke, D. Estrin, M. Hansen, E. Howard, R. West, and P. Boda, "PEIR, the personal environmental impact report, as a platform for participatory sensing systems research," in Mobile Systems, Applications and Services, 2009, pp. 55-68.

[3] R. Ben Messaoud and Y. Ghamri-Doudane, "QoI and energy-aware mobile sensing scheme: A tabu-search approach," in IEEE 82nd Vehicular Technology Conference: VTC2015-Fall, 2015.

\section{NOTES}

${ }^{1}$ Funf: http://www.funf.org/

${ }^{2}$ APISENSE online: http://www.apisense.com/

${ }^{3}$ Sensortag: http://www.ti.com/sensortag2015/ 